\title{
TGF- $\beta 1$ is Involved in Vitamin D-Induced Chondrogenic Differentiation of Bone Marrow-Derived Mesenchymal Stem Cells by Regulating the ERK/JNK Pathway
}

\author{
Xiaorui Jiang ${ }^{\mathrm{a}}$ Botao Huang ${ }^{\mathrm{a}}$ Huiying Yang ${ }^{\mathrm{b}}$ Guishi Li $^{\mathrm{a}} \quad$ Chunlei Zhang ${ }^{\mathrm{c}}$ \\ Guangshi Yang ${ }^{a}$ Feng Lin ${ }^{a}$ Guodong Lina
}

aDepartment of Orthopedics, The Affiliated Yantai Yuhuangding Hospital of Qingdao University, Yantai, ${ }^{b}$ Department of Hepatology, Yantai Infectious Disease Hospital, Yantai, 'Binzhou Medical University, Yantai, China

\section{Key Words}

Osteoarthritis $• \mathrm{BMSC} \cdot$ Vitamin D $・$ TGF- $\beta 1 \cdot$ Chondrogenic differentiation $・$ ERK/JNK pathway

\begin{abstract}
Background/Aims: Osteoarthritis (OA) is characterized by degradation of cartilage, sole cell type of which is chondrocytes. Bone marrow-derived mesenchymal stem cells (BMSCs) possess multipotency and can be directionally differentiated into chondrocytes under stimulation. This study was aimed to explore the possible roles of vitamin $D$ and transforming growth factor- $\beta 1$ (TGF- $\beta 1$ ) in the chondrogenic differentiation of BMSCs. Methods: BMSCs were isolated from femurs and tibias of rats and characterized by flow cytometry. After stimulation with vitamin $D$, BMSC proliferation and migration were measured by Cell Counting Kit-8 (CCK-8) and Transwell assays, respectively. Chondrogenic differentiation was estimated through expression levels of specific markers by qRT-PCR and Western blot analysis. After stable transfection, the effects of aberrantly expressed TGF- $\beta 1$ on vitamin D-induced alterations, including BMSC viability, migration and chondrogenic differentiation, were all evaluated utilizing CCK-8 assay, Transwell assay, qRT-PCR and Western blot analysis. Finally, the phosphorylation levels of key kinases in the extracellular signal-regulated kinase (ERK) and c-Jun N-terminal kinase (JNK) pathways were determined by Western blot analysis. Results: Vitamin D remarkably promoted BMSC viability, migration and chondrogenic differentiation. These alterations of BMSCs induced by vitamin $D$ were reinforced by TGF- $\beta 1$ overexpression while were reversed by TGF- $\beta 1$ silencing. Additionally, the phosphorylation levels of ERK, JNK and c-Jun were enhanced by TGF- $\beta 1$ overexpression but were reduced by TGF- $\beta 1$ knockdown. Conclusion: Vitamin D promoted BMSC proliferation, migration and chondrogenic differentiation. TGF- $\beta 1$ might be implicated in the vitamin D-induced alterations of BMSCs through regulating ERK/JNK pathway.
\end{abstract}




\section{Introduction}

As the most prevalent form of arthritis, osteoarthritis $(\mathrm{OA})$ is defined as degradation of articular cartilage and bone sclerosis of articular joints [1, 2]. On the basis of etiology, OA is a multifactorial disease resulting from obesity, aging, repetitive injury and abnormal loading [3]. With the increase of obesity and the ageing population, the economic and social burden focused on OA have been increased obviously in the United States [4]. Patients suffering from $\mathrm{OA}$ are companied by joint pain and physical disability throughout the life cycle, making the need of effective treatment for OA become pressing [5].

Accumulating evidence characterizes $\mathrm{OA}$ as degradation of cartilage, which is induced by multiple pathological damages to chondrocytes, the unique cell type in cartilage $[6,7]$. The apoptosis of chondrocytes is widely considered to play a crucial role in the pathogenesis of OA [8]. Bone marrow-derived mesenchymal stem cells (BMSCs) possess pluripotency during several passages, and are commonly acceptable for differentiation into many cell types, such as chondrocytes and osteoblasts [9-11]. Transplantation of BMSCs has been widely studied as promising treatments for ischemia-induced pulmonary injury [12], pelvic floor dysfunction [13] and olfactory disorder [14]. In terms of orthopaedic researches, BMSCs are proved to facilitate cartilage regeneration because of the rapid cell proliferation, multipotency and easy isolation [15]. Therefore, more detailed mechanism of the directional BMSC differentiation into chondrocytes has great significance for restoration of OA-induced cartilage injury.

The directionally inductive conditions for the chondrogenic differentiation of BMSCs into chondrocytes remain controversial. Vitamin D is a pivotal mediator of bone metabolism and calcium homeostasis through interaction with vitamin D receptor, along with notable promotion of cellular differentiation and inhibition of cell proliferation [16]. It has been reported to accelerate osteoblastic differentiation of mesenchymal stem cells [17], whereas its role in chondrogenic differentiation has not yet been well studied. Transforming growth factor- $\beta 1$ (TGF- $\beta 1$ ) is a growth factor involved in cell differentiation, proliferation and migration [18]. A previous study once demonstrated TGF- $\beta 1$ could induce cardiomyogenic differentiation of BMSCs. Another study illustrated that differentiation of BMSCs into chondrocytes could be induced by synergistic actions of TGF- $\beta 1$ stimulation and 3D culture system [19]. Nevertheless, the interactions of vitamin D and TGF- $\beta 1$ in the chondrogenic differentiation of BMSCs remain unclear.

In our study, we aimed to figure out the functional roles of vitamin D in cell proliferation, migration and chondrogenic differentiation of BMSCs. The involvements of TGF- $\beta 1$ in the modulation of vitamin D had been also studied. Furthermore, the possible molecular mechanism was investigated simultaneously.

\section{Materials and Methods}

\section{Animal and ethics statements}

Two-week-old male Sprague-Dawley (SD) rats were provided by the Liaocheng People's Hospital. Rats were housed under clean conventional conditions $\left(21-25^{\circ} \mathrm{C}\right.$, a humidity of $45-50 \%$ and a 12 -h light/dark cycle) with pellet chow and water freely available. All the experimental procedures were approved by the Ethics Committee of The Affiliated Yantai Yuhuangding Hospital of Qingdao University.

\section{Primary BMSC culture and vitamin D preparation}

Primary BMSCs were acquired as described previously [20]. In brief, the femur and tibias of SD rats were carefully separated and washed with phosphate-buffered saline (PBS). Then, the bone marrow was obtained from the cavities of these bones, followed by cell culture in Iscove's Modified Dulbecco's Medium (IMDM) containing 10\% fetal bovine serum (FBS), $100 \mathrm{U} / \mathrm{mL}$ penicillin and $100 \mu \mathrm{g} / \mathrm{mL}$ streptomycin (all from Invitrogen, Carlsbad, CA, USA). Cells were maintained in a humidified incubator with $5 \% \mathrm{CO}_{2}$ at $37^{\circ} \mathrm{C}$. 


\section{Cellular Physiology Cell Physiol Biochem 2017;42:2230-2241 \begin{tabular}{l|l|l} 
and Biochemistry & DOI:1159/000479997 & $\begin{array}{l}\text { C) } 2017 \text { The Author(s). Published by S. Karger AG, Basel } \\
\text { www.karger.com/cpb }\end{array}$
\end{tabular}}

Jiang et al.: Roles of Vitamin D and TGF- $\beta 1$ in BMSCs

After $3 \mathrm{~d}$ of incubation, the culture media was changed to remove non-adherent cells. The adherent cells were rinsed with PBS and cultured along with medium exchange every $3 \mathrm{~d}$ until the confluence reached 80$90 \%$. Thereafter, cells were passaged at 1:3 and cultured again. Passage 3 cells were used for subsequent experiments.

The characterization of BMSCs was performed through analysis of typical surface markers using flow cytometry. BMSCs (passage 3, $1 \times 10^{6}$ cells) were collected, washed and incubated with antibodies against CD29, CD90, CD45 or CD105 (all from BD Biosciences, San Diego, CA, USA) according to the manufacturer's instructions, followed by flow cytometry analysis using a BD $^{\mathrm{TM}}$ LSR II Flow Cytometer System (BD Biosciences).

$1 \alpha, 25(\mathrm{OH})_{2} \mathrm{D}_{3}$ (vitamin D; Sigma-Aldrich, St. Louis, MO, USA) was dissolved in dimethyl sulfoxide (DMSO) with a final concentration of $100 \mu \mathrm{M}$, acting as stock solution. The concentration of vitamin D for further studies was $10 \mathrm{nM}$ or $100 \mathrm{nM}$ in culture medium.

Cell Counting Kit-8 (CCK-8) assay

Cell viability was evaluated by CCK- 8 assay following the instructions of supplier. Briefly, cells $\left(5 \times 10^{3}\right.$ cells/well) were seeded in a 96-well plate and treated differently. Then, $10 \mu \mathrm{L}$ of CCK-8 solution (Dojindo Molecular Technologies, Gaithersburg, MD, USA) was added into each well and the mixture was incubated for $1 \mathrm{~h}$ at $37^{\circ} \mathrm{C}$ with $5 \% \mathrm{CO}_{2}$. The absorbance was read using a Microplate Reader (Bio-Rad, Hercules, CA, USA) at $450 \mathrm{~nm}$.

\section{Transwell assay}

Cell migration was examined by $8-\mu \mathrm{m}$ pore Transwell filter system (BD Biosciences). The lower chamber of the system was filled with $600 \mu \mathrm{L}$ complete medium while the upper chamber was filled with $200 \mu \mathrm{l}$ of BMSCs suspended in serum-free medium. The Transwell filter system containing BMSCs were then subjected into a humidity incubator. After $12 \mathrm{~h}$ of incubation at $37^{\circ} \mathrm{C}$, BMSCs were fixed with methanol and the non-traversed cells staying on the upper surface of the filter were removed carefully with a cotton swab. Migrated (traversed) cells on the lower side of the filter were stained with crystal violet for $15 \mathrm{~min}$, followed by counting in five randomly selected fields under an inverted microscope (Olympus, Tokyo, Japan).

\section{Chondrogenic induction of BMSCs in vitro}

The chondrogenic differentiation of BMSCs in vitro was induced as described previously [21]. Briefly, BMSCs $\left(1 \times 10^{5}\right.$ cells/well) were plated onto 24 -well plates and incubated at $37^{\circ} \mathrm{C}$ until the confluence reached $80 \%$. Then, the culture medium of BMSCs was replaced by chondrogenic-inductive medium in the presence or absence of vitamin $\mathrm{D}$. The composition of chondrogenic-inductive medium was identical to a previous literature [21]. The induction of chondrogenic differentiation of BMSCs was persisted for $14 \mathrm{~d}$, and then the resulted BMSCs were collected for quantitative reverse transcription PCR (qRT-PCR) or Western blot analysis.

\section{Cell transfection and generation of stably transfected cell lines}

Short-hairpin RNA (shRNA) targeting rat TGF- $\beta 1$ was ligated into the U6/GFP/Neo plasmid (GenePharma, Shanghai, China) to construct sh-TGF- $\beta 1$. The U6/GFP/Neo plasmid carrying a non-targeting sequence (sh-NC) was acted as the negative control of sh-TGF- $\beta 1$. The full-length of rat TGF- $\beta 1$ sequences was sub-cloned into the pEX-2 plasmid (GenePharma) to construct pEX-TGF- $\beta 1$. The empty pEX-2 plasmid was acted as the negative control of pEX-TGF- $\beta 1$. These three recombined plasmids and empty pEX-2 were respectively transfected into BMSCs by using lipofectamine 3000 reagent (Life Technologies Corporation, Carlsbad, CA, USA) according to the manufacturer's instructions. After selection with $0.5 \mathrm{mg} / \mathrm{mL}$ G418 (Sigma-Aldrich) for approximately 4 weeks, stably transfected BMSCs were successfully obtained.

qRT-PCR

After treatments, TRIzol reagent (Invitrogen) and DNaseI (Promega, Madison, WI, USA) were used for the isolation of total RNA in BMSCs. Complementary DNA (cDNA) was synthesized by reverse transcription using the Multiscribe RT kit (Applied Biosystems, Foster City, CA, USA) and random hexamers or oligo(dT) in line with the program: $10 \mathrm{~min}$ at $25^{\circ} \mathrm{C}, 30 \mathrm{~min}$ at $48^{\circ} \mathrm{C}$, and a final step of $5 \mathrm{~min}$ at $95^{\circ} \mathrm{C}$. Then, quantitative PCR was performed using SYBR Green Master Mix (Applied Biosystems) following the supplier's protocol. 


\section{Cellular Physiology Cell Physiol Biochem 2017;42:2230-2241

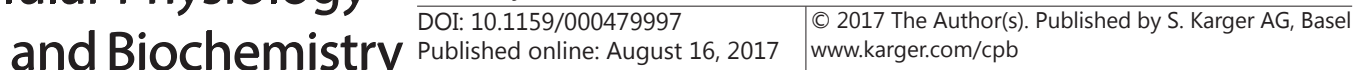 \\ Jiang et al.: Roles of Vitamin D and TGF- $\beta 1$ in BMSCs}

The primers used for quantitative PCR were designed and synthesized by GenScript Co., Ltd. (Nanjing, China). Relative mRNA expression was calculated on the basis of $2^{-\Delta \Delta C t}$ method [22], and the results were normalized to GAPDH.

\section{Western blot analysis}

After treatments, RIPA lysis buffer (Beyotime Biotechnology, Shanghai, China) supplemented with Halt protease and phosphatase inhibitor cocktail (Pierce, Rockford, IL, USA) was used for the extraction of protein in BMSCs. After quantification with $\mathrm{BCA}^{\mathrm{TM}}$ Protein Assay Kit (Pierce), equivalent proteins were separated by sodium dodecyl sulfate-polyacrylamide gel electrophoresis (SDS-PAGE). Then, proteins in the gels were transferred to polyvinylidene difluoride (PVDF) membrane, and the membranes were blocked with non-fat milk at room temperature for $1 \mathrm{~h}$. Primary antibodies against Sry-related high mobility group box 9 (Sox9, ab185230), Collagen II (Col-II, ab188570), Aggrecan (ab36861), Collagen X (Col-X, ab182563), TGF- $\beta 1$ (ab179695), extracellular signal-regulated kinase (ERK, ab184699), phosphorylated ERK (p-ERK, ab201015), c-Jun N-terminal kinase (JNK, ab199380), phosphorylated JNK (p-JNK, ab47337), c-Jun (ab32137), phosphorylated c-Jun (p-c-Jun, ab32385) or GAPDH (ab9484) were incubated with the membrane at $4^{\circ} \mathrm{C}$ overnight, respectively. Subsequently, the membranes were washed and incubated with secondary antibody conjugated to horseradish peroxidase (HRP) for $1 \mathrm{~h}$ at room temperature. After being washed again, the membranes were subjected to the Bio-Rad ChemiDoc ${ }^{\mathrm{TM}}$ XRS system, and $200 \mu \mathrm{L}$ of Immobilon Western Chemiluminescent HRP Substrate (Millipore, Billerica, MA, USA) was added. The signals were captured and analyzed by using Image Lab ${ }^{\mathrm{TM}}$ software (Bio-Rad).

\section{Statistical analysis}

All experiments were repeated three times. The results are presented as the mean \pm standard error of the mean (SEM). Statistical analysis was performed using Graphpad Prism 5 software (GraphPad, San Diego, CA, USA). The $P$-values were calculated using the two-way analysis of variance (ANOVA) with Bonferroni's correction or multiple $t$-tests. A $P$-value of $<0.05$ was considered to indicate a statistically significant result.

\section{Results}

\section{Characterization of BMSCS}

To verify whether these cells we obtained were BMSCs, flow cytometry analysis was performed. We explored the expressions of three BMSCs-specific molecular including CD29, CD90 and CD105, and the expression of haematopoietic marker CD45. In Fig. 1, the cells we isolated exhibited strong positive signals for CD29+, CD90+ and CD105 ${ }^{+}$(Fig. 1A-1C), whereas these cells were negative for CD45- (Fig. 1D). All these results suggested that the cells we obtained were BMSCs.

Vitamin D promotes BMSC proliferation and migration

Cell viability and migration were both estimated to explore the effect of vitamin D on BMSCs. After stimulation with different doses of vitamin D (0,10 or $100 \mathrm{nM})$, cell viability was markedly increased by $100 \mathrm{nM}$ of vitamin D compared with cells treated with $0 \mathrm{nM}$ of vitamin $\mathrm{D}(P<0.05$, Fig. $2 \mathrm{~A})$, whereas the influence of $10 \mathrm{nM}$ vitamin $\mathrm{D}$ was not significant. Analogically, cell migration was also markedly enhanced by $100 \mathrm{nM}$ vitamin D compared with cells treated with $0 \mathrm{nM}$ of vitamin $\mathrm{D}(P<0.05$, Fig. $2 \mathrm{~B})$, along with not obviously significant influence of $10 \mathrm{nM}$ vitamin D. The results illustrated that vitamin D promoted BMSC proliferation and migration.

Vitamin D promotes chondrogenic differentiation of BMSCs

BMSCs were stimulated with 0,10 or $100 \mathrm{nM}$ vitamin $\mathrm{D}$, and the expressions of chondrogenic differentiation-associated genes and proteins were determined at $3 \mathrm{~d}, 7$ $\mathrm{d}$ and $14 \mathrm{~d}$ after stimulation. The results in Fig. 3A-3D showed mRNA levels of Sox9, type II procollagen (Col2a1), Aggrecan and type X procollagen (Col10a1) were all remarkably increased by $10 \mathrm{nM}$ or $100 \mathrm{nM}$ vitamin D compared with non-treated cells at $7 \mathrm{~d}$ and 14 
Fig. 1. Identity and purity of bone marrow-derived mesenchymal stem cells (BMSCs). The isolated BMSCs were incubated with FITC labeled antibodies against CD29, CD90, CD105 or CD45, followed by assessments of flow cytometry. These isolated cells were positive for the stem cell markers CD29+ (A), CD90+ (B) and $\mathrm{CD} 105^{+}(\mathrm{C})$ and were negative for the hematopoietic stem cell marker CD45- (D).

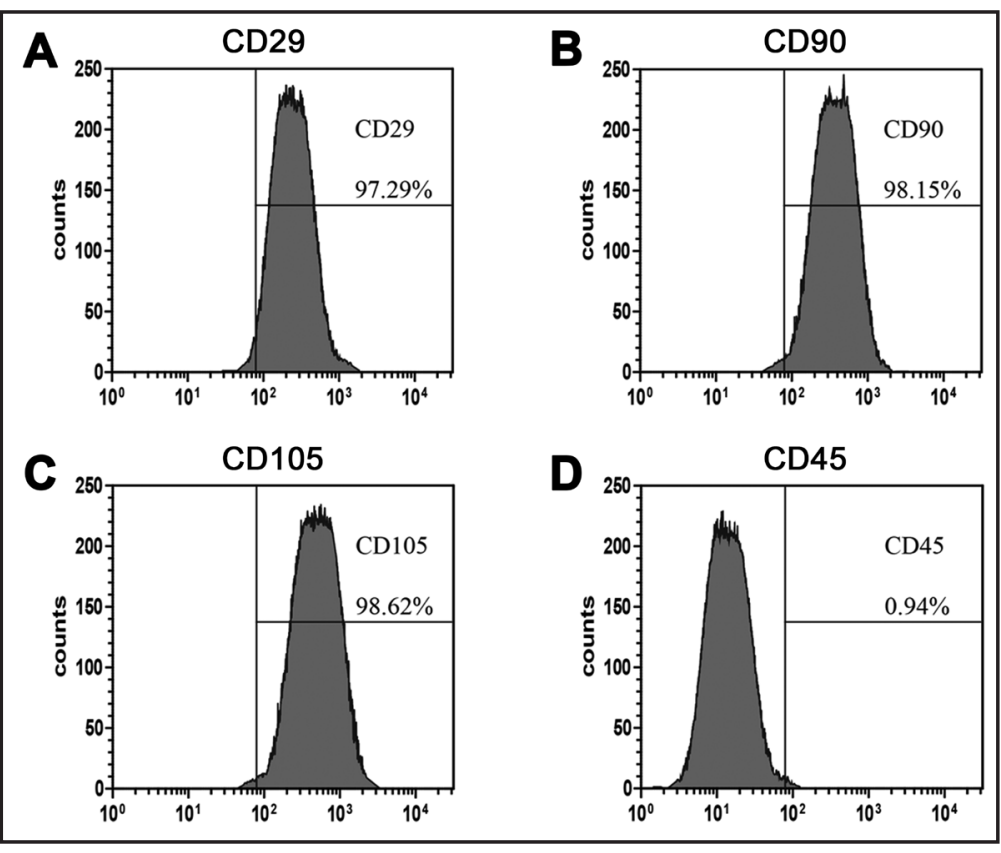

Fig. 2. Vitamin D promotes BMSC proliferation and migration. BMSCs were stimulated with different doses of vitamin D (0, 10 and $100 \mathrm{nM})$. (A) Cell viability was measured by a Cell Counting Kit-8 assay. (B) Cell migration was measured by a Transwell assay. Data are presented as the mean \pm standard error of the mean (SEM). ${ }^{*}, P<0.05$. BMSCs, bone marrow-derived mesenchymal stem cells.

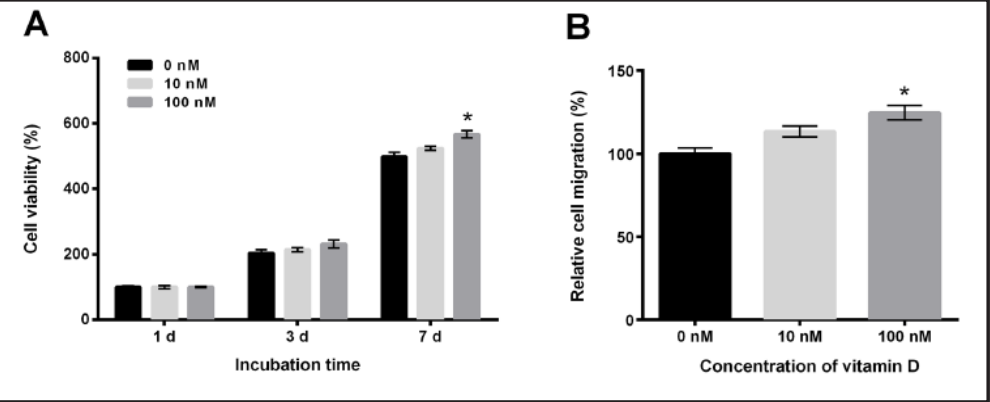

A

B

$\mathrm{d}$ after induction $(P<0.05$ or $P<0.001)$. The influence of vitamin on protein expressions of Sox9, Col-II, Aggrecan and Col-X were consistent with corresponding genes, presenting obvious enhancements of protein expression in vitamin D-induced BMSCs. Collectively, we drew a conclusion that vitamin D could promote chondrogenic differentiation of BMSCs.

TGF- $\beta 1$ participates in the vitamin D-induced promotion of BMSC proliferation and migration

Three recombined plasmids and empty pEX-2 were respectively transfected into BMSCs in order to stably overexpress or silence TGF- $\beta 1$. Results in Fig. 4A stated mRNA and protein expression levels of TGF- $\beta 1$ were both observably elevated in cells transfected with pEX-TGF- $\beta 1$ compared with pEX-2 group $(P<0.01)$ while were both remarkably reduced in cells transfected with sh-TGF- $\beta 1$ compared with sh-NC group $(P<0.01)$. The results demonstrated that aberrant expression of TGF- $\beta 1$ was successfully realized in BMSCs. Then, non-transfected and transfected cells were all stimulated by $10 \mathrm{nM}$ vitamin $\mathrm{D}$, followed by assessment of cell viability and migration. When compared to cells transfected with pEX-2, cell viability was significantly increased by TGF- $\beta 1$ overexpression at $3 \mathrm{~d}$ and $7 \mathrm{~d}$ (both $P$ $<0.05$, Fig. 4B) after vitamin D stimulation. Likewise, the effect of TGF- $\beta 1$ overexpression on cell migration was the same as cell viability, showing a significant elevation in TGF- $\beta 1$ overexpressed BMSCs $(P<0.05$, Fig. 4C). However, the influence of TGF- $\beta 1$ knockdown on 
A
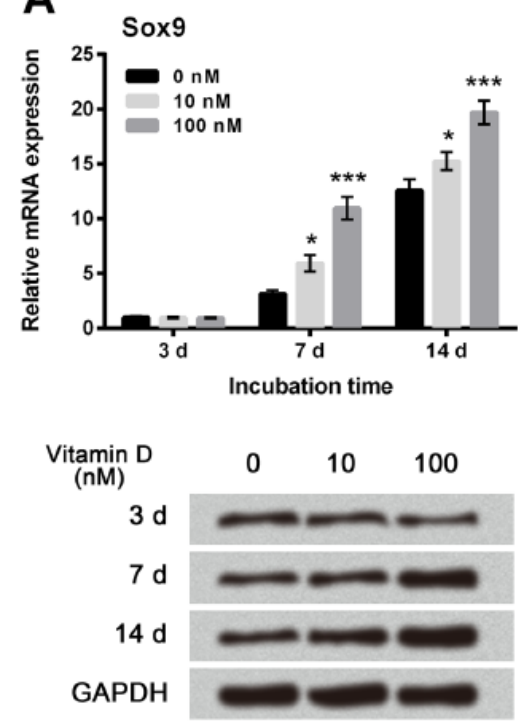

C

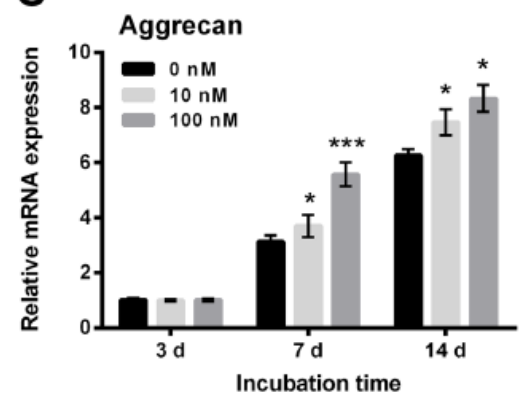

Vitamin D

(nM)

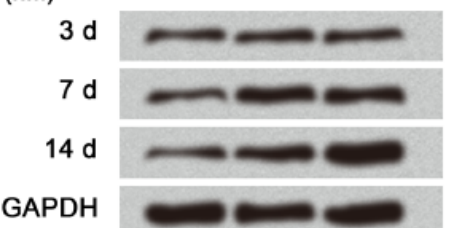

B
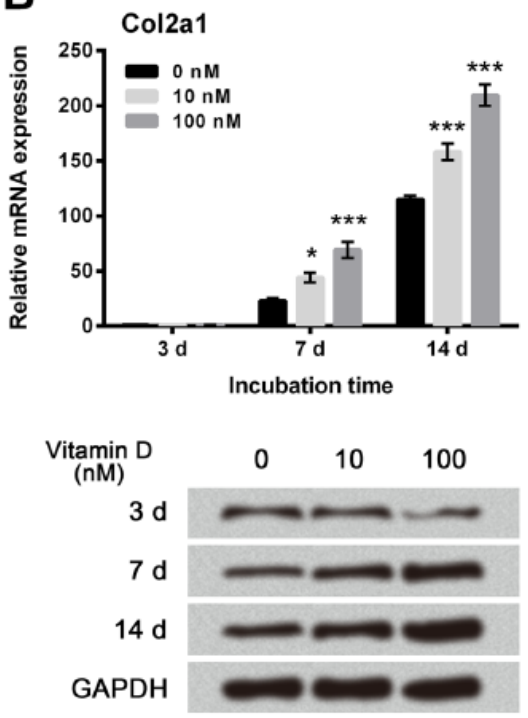

D
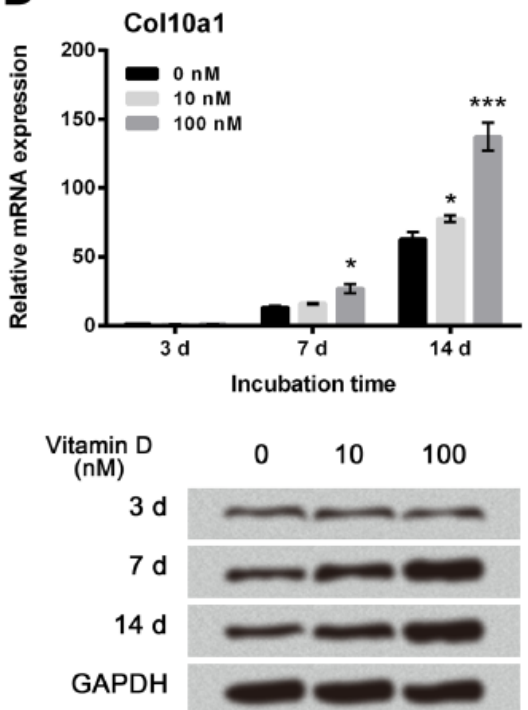

Fig. 3. Vitamin D promotes chondrogenic differentiation of BMSCs. BMSCs were stimulated with different doses of vitamin D (0,10 and $100 \mathrm{nM})$ and cultured for 14 days. mRNA and protein expression levels were assessed at $3 \mathrm{~d}, 7 \mathrm{~d}$ and $14 \mathrm{~d}$ after vitamin D stimulation by qRT-PCR and Western blot analysis, respectively. BMSCs treated with $0 \mathrm{nM}$ of vitamin D were acted as control. (A) mRNA and protein levels of Sox9. (B) mRNA level of Col2a1 and protein level of collagen II. (C) mRNA and protein levels of Aggrecan. (D) mRNA level of Col10a1 and protein level of collagen X. Data are presented as the mean \pm standard error of the mean (SEM). *, $P<0.05$; **, $P<0.001$. BMSCs, bone marrow-derived mesenchymal stem cells; Sox9, Sry-related high mobility group box 9; Col2a1, type II procollagen; Col10a1, type X procollagen.

cell viability and migration of BMSCs after vitamin D stimulation was opposite to TGF- $\beta 1$ overexpression. All the results suggested that TGF- $\beta 1$ was involved in the vitamin D-induced alteration of cell proliferation and migration. 
Fig. 4. TGF- $\beta 1$ is involved in the vitamin D (VD)-induced promotion of BMSC proliferation and migration. BMSCs were transfected with pEX-2, pEX-TGF- $\beta 1$, sh-NC or sh-TGF- $\beta 1$ and selected by G418. (A) mRNA and provels of TGF- $\beta 1$ were assessed by qRT-PCR and Western blot analysis, respectively. (B) Cell viability was measured by a Cell Counting Kit-8 assay after stimulation of $10 \mathrm{nM}$ VD. (C) Cell migration was measured by a Transwell assay after stimulation of $10 \mathrm{nM}$ VD. the mean \pm standard error of the mean (SEM). *, $P<0.05$; **, $P<0.01$; ***, $P<$ 0.001. BMSCs, bone m a r r ow-derived mesenchymal stem cells; TGF- $\beta 1$, transforming growth factor- $\beta 1$; $\quad$ EXX-TGF- $\beta 1$, pEX-2 plasmid containing full length of tein expression leData are presented as

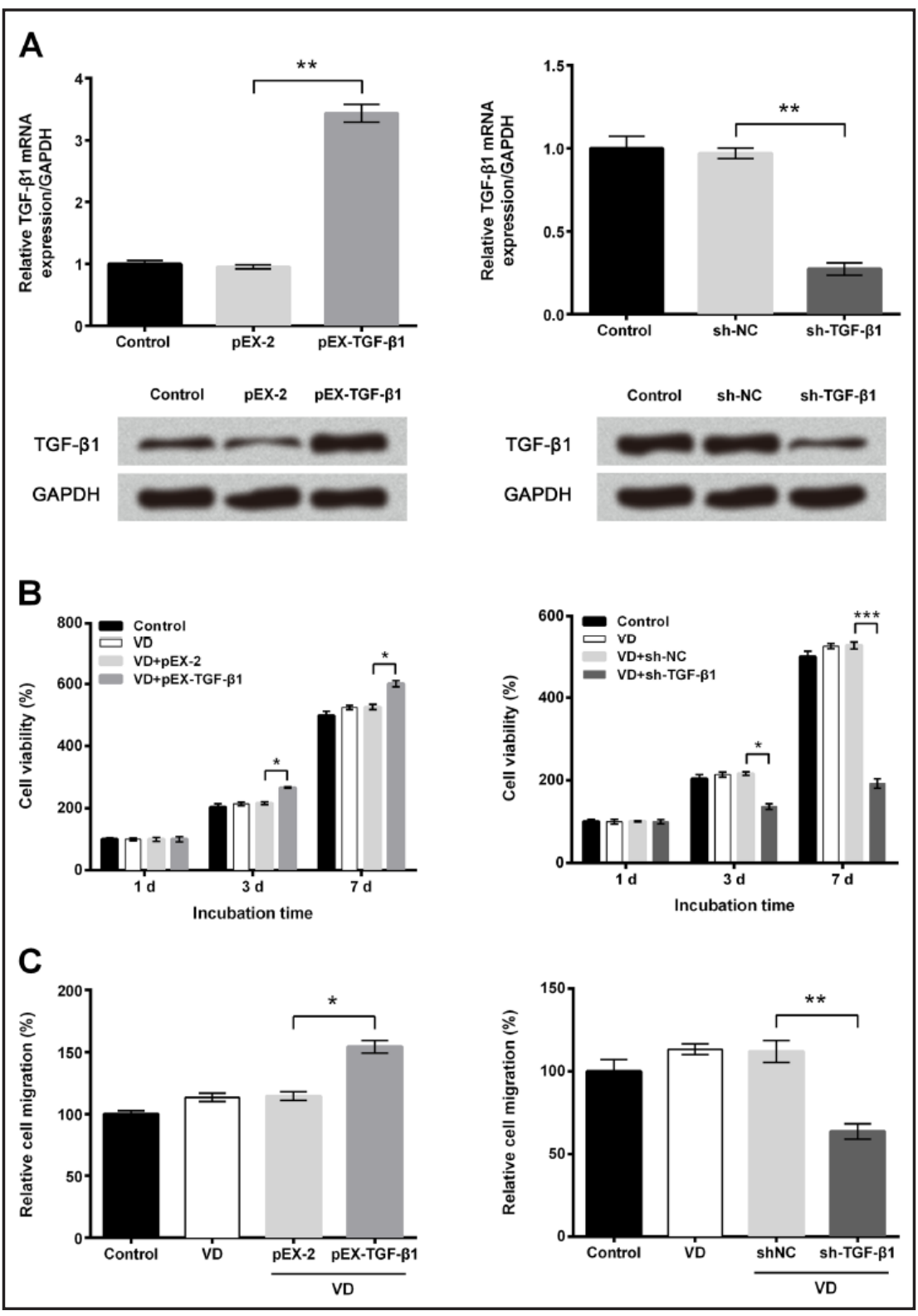

rat TGF- $\beta 1$; sh-TGF- $\beta 1$, U6/GFP/Neo plasmid containing short-hairpin RNA against rat TGF- $\beta 1$; sh-NC, U6/ GFP/Neo plasmid containing a non-targeting sequence.

TGF- $\beta 1$ participates in the vitamin D-induced chondrogenic differentiation of BMSCs

To further explore the involvements of TGF- $\beta 1$ in the vitamin D-induced chondrogenic differentiation, transfected and non-transfected BMSCs were stimulated with vitamin D for 14 days. As shown in Fig. 5A-5B, Sox9, Cola2a1, Aggrecan and Col10a1 were all prominently up-regulated by TGF- $\beta 1$ overexpression $(P<0.05$ or $P<0.01)$ while were notably downregulated by TGF- $\beta 1$ silence $(P<0.05$ or $P<0.01)$ at $7 \mathrm{~d}$ and $14 \mathrm{~d}$ of vitamin D stimulation when compared to respective controls. Therefore, we concluded that TGF- $\beta 1$ was involved in the vitamin D-induced chondrogenic differentiation of BMSCs.

TGF- $\beta 1$ activates ERK/JNK signaling pathway

To reveal the underlying mechanisms of TGF- $\beta 1$-associated regulations, the phosphorylation of key kinases involved in ERK/JNK signaling pathway was evaluated 


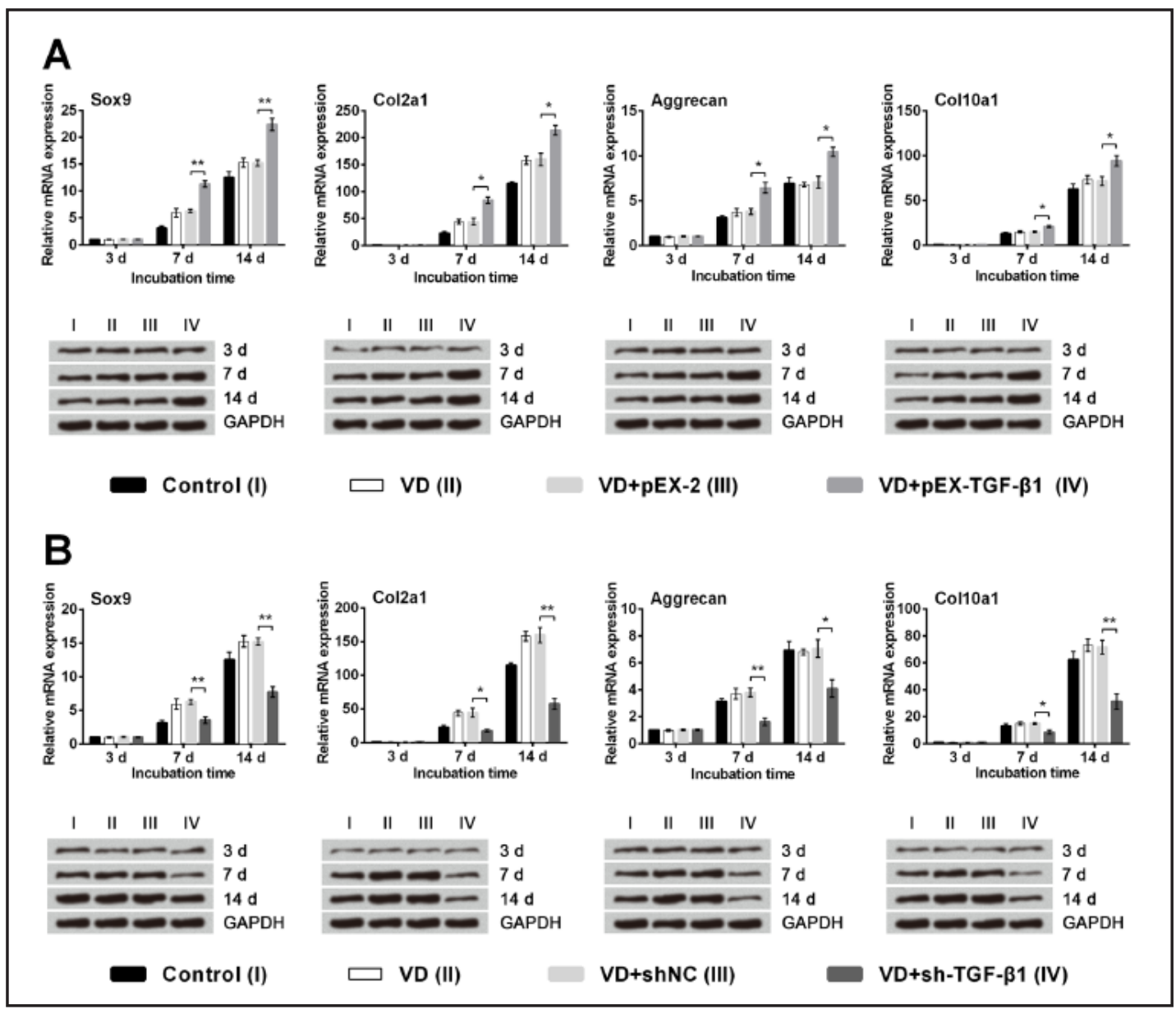

Fig. 5. TGF- $\beta 1$ is involved in the vitamin D (VD)-induced chondrogenic differentiation of BMSCs. BMSCs were transfected with pEX-2, pEX-TGF- $\beta 1$, sh-NC or sh-TGF- $\beta 1$ and selected by G418. mRNA and protein expression levels were assessed at $3 \mathrm{~d}, 7 \mathrm{~d}$ and $14 \mathrm{~d}$ after $10 \mathrm{nM}$ of VD stimulation by qRT-PCR and Western blot analysis, respectively. mRNA and protein levels of chondrogenic differentiation specific markers were all up-regulated by TGF- $\beta 1$ overexpression (A) but were down-regulated by TGF- $\beta 1$ knockdown (B) under VD stimulation. Data are presented as the mean \pm standard error of the mean (SEM). ${ }^{*}, P<0.05 ;{ }^{* *}, P<$ 0.01 . BMSCs, bone marrow-derived mesenchymal stem cells; TGF- $\beta 1$, transforming growth factor- $\beta 1$; $p E X-$ TGF- $\beta 1$, pEX-2 plasmid containing full length of rat TGF- $\beta 1$; sh-TGF- $\beta 1$, U6/GFP/Neo plasmid containing short-hairpin RNA against rat TGF- $\beta 1$; sh-NC, U6/GFP/Neo plasmid containing a non-targeting sequence; Sox9, Sry-related high mobility group box 9; Col2a1, type II procollagen; Col10a1, type X procollagen.

in BMSCs with abnormal expression of TGF- $\beta 1$. Western blot analysis in Fig. 6 illustrated phosphorylated levels of ERK, JNK and c-Jun were all obviously enhanced by TGF- $\beta 1$ overexpression while were observably reduced by TGF- $\beta 1$ knockdown, suggesting that TGF- $\beta 1$ could activate ERK/JNK signaling pathway.

\section{Discussion}

BMSCs are a kind of multipotency cells which could be directly induced to differentiate into various cells types, including chondrocytes. As the critical and sole cell type in cartilage, chondrocytes are considered to affect progression of OA tightly. Thus, the study on the directional differentiate from BMSCs into chondrocytes is meaningful for the therapy of cartilage degradation in OA. Herein, in our study, we successfully isolated rat BMSCs 
Fig. 6. TGF- $\beta 1$ regulates activation of ERK/ JNK signaling pathway in BMSCs. BMSCs were transfected with pEX-2, pEX-TGF- $\beta 1$, sh-NC or sh-TGF- $\beta 1$ and selected by G418. Phosphorylation of key kinases involved in ERK and JNK pathways were assessed by Western blot analysis. BMSCs, bone marrow-derived mesenchymal stem cells; TGF- $\beta 1$, transforming growth factor- $\beta 1$; $p$ EX-TGF- $\beta 1, p E X-2$ plasmid containing full length of rat TGF- $\beta 1$; sh-TGF- $\beta 1$, U6/GFP/Neo plasmid containing short-hairpin RNA against rat TGF- $\beta 1$; sh-NC, U6/GFP/Neo plasmid containing a non-targeting sequence; ERK, extracellular signal-regulated kinase; JNK, c-Jun N-terminal kinase.

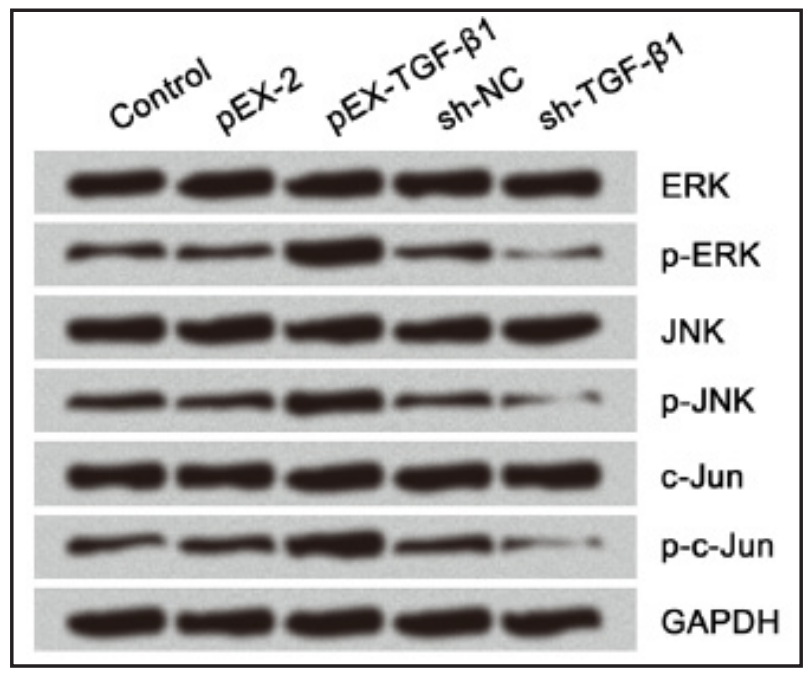

and interestingly identified $100 \mathrm{nM}$ of vitamin D markedly promoted cell proliferation and migration of BMSCs. Further studies illustrated vitamin D promoted chondrogenic differentiation of BMSCs. Subsequently, we abnormally expressed TGF- $\beta 1$ and found the effects of vitamin D on BMSCs were reinforced by TGF- $\beta 1$ overexpression while were significantly decreased by TGF- $\beta 1$ knockdown. The final Western blot analysis indicated aberrant expression of TGF- $\beta 1$ influenced the activation of ERK/JNK signaling pathway.

BMSCs are characterized by the presence of antigens such as CD29, CD90 and CD105 and by the absence of CD45 [23], thus we performed flow cytometry to identify whether the cells we isolated were BMSCs first of all using the corresponding antibodies. The results of flow cytometry presenting the obvious $\mathrm{CD} 29^{+}, \mathrm{CD}^{\circ} 0^{+}, \mathrm{CD} 105^{+}$and $\mathrm{CD} 45^{-}$confirmed that the isolated cells were BMSCs. During the development of cartilage, local mesenchymal cells were observed to aggregate, suggesting the importance of enough cell density for the differentiation to cartilage [24]. A previous literature has proved that BMSCs differentiate into chondrocytes when the number of cells increased to a certain level [25]. Meanwhile, BMSCs could migrate to the damaged cartilage followed by differentiation into chondrocytes, and thereby are deemed to act as good cell sources for the regeneration of cartilage [26]. Thus, cell proliferation and migration of BMSCs were major factors for the chondrogenic differentiation. In our study, we stimulated BMSCs with different doses of vitamin D and discovered cell proliferation and migration were both obviously promoted under stimulation of $100 \mathrm{nM}$ vitamin D. However, the impact on cell proliferation and migration was slight under stimulation of $10 \mathrm{nM}$ vitamin $\mathrm{D}$, along with non-significant significance.

Sox9 is a member of Sox family and its mutation leads to skeletal malformation syndrome campomelic dysplasia [27]. Expression of Sox9 starts from mesenchymal progenitor cells and is increased in chondroprogenitors and chondrocytes [28]. Sox9 mRNA is required for the activation of Col2a1 transcription, and the parallel expressions of Sox 9 and Col2a1 were observed in cells undergoing chondrogenesis [29]. The expression of another collagen gene, Col10a1, is also reported to be activated during chondrogenesis of MSCs [30]. Aggrecan is the main part of proteoglycans synthesized by chondrocytes [31]. Thus, these four factors are commonly considered as specific markers for chondrocytes. In our study, we evaluated the mRNA expression levels of Sox9, Col2a1, Aggrecan and Col10a1 along with protein expression levels of Sox9, Col-II, Aggrecan and Col-X, and then discovered these factors were all significantly up-regulated by vitamin D stimulation, suggesting an obvious elevation of chondrogenic differentiation.

A previous literature has mentioned that TGFs are effective in prevention of dedifferentiation of chondrocytes and promotion of cell growth [32]. Thus, we further explored the potential involvements of TGF- $\beta 1$ in the vitamin D-mediated enhancements of BMSC proliferation, migration and differentiation. Accordingly, we constructed stably transfected BMSCs, leading to abnormal expression of TGF- $\beta 1$. After stimulation of vitamin 
$\mathrm{D}$, results showed that the non-significant influence of vitamin $\mathrm{D}(10 \mathrm{nM})$ on BMSC proliferation and migration was markedly reinforced in TGF- $\beta 1$ overexpressed cells while was surprisingly reduced in TGF- $\beta 1$ silenced cells. Moreover, the extent of the reduction was much larger than reinforcement, indicating that the effects of vitamin D on BMSCs might be affected dramatically by TGF- $\beta 1$ expression. Subsequent experiments of specific markers of chondrogenic differentiation also consolidated the speculation, showing intensive influence of vitamin D in TGF- $\beta 1$ overexpressed BMSCs but converse influence in TGF- $\beta 1$ silenced BMSCs.

Mitogen-activated protein kinases (MAPKs), comprised of JNK, p38 and ERK, are serine-threonine protein kinases and participate in a myriad of cellular activities, such as proliferation, inflammation, migration and differentiation [33]. JNK signaling pathway has been reported to participate in differentiation from MSC into cardiocytes under stimulation of Cyclooxygenase 2 [34]. Wang et al. also proved that activation of ERK and JNK pathways are involved in the pyruvate dehydrogenase kinase isoform 2-induced chondrogenic differentiation of MSCs. To elucidate the possible underlying mechanism, we finally evaluated the phosphorylation of key kinases involved in ERK and JNK pathways in stably transfected BMSCs. Results illustrated that TGF- $\beta 1$ overexpression observably activated these two pathways, suggesting that TGF- $\beta 1$ might participate in the modulation of vitamin D through regulating ERK and JNK pathways in BMSCs. Taken together, we discovered vitamin D could promote BMSC proliferation and migration and play a potential role in the directionally chondrogenic differentiation of BMSCs. TGF- $\beta 1$ expression was involved in the functional roles of vitamin D through regulation of ERK/JNK signaling pathways. The results in our study are of great importance for the application of BMSCs on the restoration of OA-induced cartilage injury. More details of the interactions between vitamin D and TGF- $\beta 1$ along with the alterations of these two signaling pathways in the chondrogenic differentiation of BMSCs will be deeply investigated in the future.

\section{Funding}

This study was supported by a program for Yantai Science and Technology Development (No. 2011208, 2011209) and Shandong Science and Technology Development (2011QW034), The National Natural Science Fund (No. 81301570).

\section{Disclosure Statement}

The authors have no conflict of interest to declare.

\section{References}

1 Bayjensen AC, Slagboom E, Chenan P, Alexandersen P, Qvist P, Christiansen C, Meulenbelt I, Karsdal MA: Role of hormones in cartilage and joint metabolism: understanding an unhealthy metabolic phenotype in osteoarthritis. Menopause 2013;20:578-586.

- Hassan AS, El-Shafey AM, Ahmed HS, Hamed MS: Effectiveness of the intra-articular injection of platelet rich plasma in the treatment of patients with primary knee osteoarthritis. Egyptian Rheumatologist 2015;37:119-124.

3 Johnson VL, Giuffre BM, Hunter DJ: Osteoarthritis: what does imaging tell us about its etiology? Semin Musculoskel Radiol 2012;16:410-418.

4 Bozic KJ, Stacey B, Berger A, Sadosky A, Oster G: Resource utilization and costs before and after total joint arthroplasty. BMC Health Serv Res 2012;12:73.

5 Bagi CM, Berryman E, Zakur DE, Wilkie D, Andresen CJ: Effect of antiresorptive and anabolic bone therapy 


\section{Cellular Physiology Cell Physiol Biochem 2017;42:2230-2241 \begin{tabular}{ll|l} 
and Biochemistry & DOI: 10.1159/000479997 & $\begin{array}{l}\text { (c) } 2017 \text { The Author(s). Published by S. Karger AG, Basel } \\
\text { www.karger.com/cpb }\end{array}$
\end{tabular}}

Jiang et al.: Roles of Vitamin D and TGF- $\beta 1$ in BMSCs

on development of osteoarthritis in a posttraumatic rat model of OA. Arthritis Res Ther 2015;17:1-16.

6 Kawasaki M, Ezura Y, Hayata T, Notomi T, Izu Y, Noda M: TGF-Beta Suppresses Ift88 Expression in Chondrocytic ATDC5 Cells. J Cell Physiol 2015;230:2788.

7 Yuan Y, Zhang GQ, Chai W, Ni M, Xu C, Chen JY: Silencing of microRNA-138-5p promotes IL-1 $\beta$-induced cartilage degradation in human chondrocytes by targeting FOXC1: miR-138 promotes cartilage degradation. Bone Joint Res 2016;5:523.

-8 Huang JG, Xia C, Zheng XP, Yi TT, Wang XY, Song G, Zhang B: 17 $\beta$-Estradiol promotes cell proliferation in rat osteoarthritis model chondrocytes via PI3K/Akt pathway. Cell Mol Biol Lett 2011;16:564-575.

-9 Pittenger MF, Mackay AM, Beck SC, Jaiswal RK, Douglas R, Mosca JD, Moorman MA, Simonetti DW, Craig S, Marshak DR: Multilineage potential of adult human mesenchymal stem cells. Science 1999;284:143-147.

10 Sun T, Sun BC, Ni CS, Zhao XL, Wang XH, Qie S, Zhang DF, Gu Q, Qi H, Zhao N: Pilot study on the interaction between B16 melanoma cell-line and bone-marrow derived mesenchymal stem cells. Cancer Lett 2008;263:35-43.

11 Tohma Y, Dohi Y, Ohgushi H, Tadokoro M, Akahane M, Tanaka Y: Osteogenic activity of bone marrowderived mesenchymal stem cells (BMSCs) seeded on irradiated allogenic bone. J Tissue Eng Regen Med 2012;6:96-102.

12 He Q, He X, Wang Y, Zou Y, Xia Q Xiong LL, Luo C, Hu X, Liu J, Wang T: Transplantation of bone marrowderived mesenchymal stem cells (BMSCs) improves brain ischemia-induced pulmonary injury in rats associated to TNF- $\alpha$ expression. Behav Brain Funct 2016;12:1-14.

13 Jin M, Ying C, Yun Z, Yan M, Wei L, Pan C, Hua X: Transplantation of bone marrow-derived mesenchymal stem cells expressing elastin alleviates pelvic floor dysfunction. Stem Cell Res Ther 2016;7:51.

14 Jo H, Jung M, Seo DJ, Park DJ: The effect of rat bone marrow derived mesenchymal stem cells transplantation for restoration of olfactory disorder. Biochem Bioph Res Commun 2015;467:395.

-15 Kagami H, Agata H, Tojo A: Bone marrow stromal cells (bone marrow-derived multipotent mesenchymal stromal cells) for bone tissue engineering: basic science to clinical translation. Int J Biochem Cell Biol 2014;20:229-232.

16 Russo I, Caroppo F, Alaibac M: Vitamins and Melanoma. Cancers 2015;7:1371-1387.

17 Piek E, Sleumer LS, van Someren EP, Heuver L, de Haan JR, De GI, Gilissen C, Hendriks JM, Ri RVO, Bauerschmidt S: Osteo-transcriptomics of human mesenchymal stem cells: accelerated gene expression and osteoblast differentiation induced by vitamin D reveals c-MYC as an enhancer of BMP2-induced osteogenesis. Bone 2010;46:613-627.

18 Zhang F, Ren T, Wu J: TGF- $\beta 1$ induces apoptosis of bone marrow-derived mesenchymal stem cells via regulation of mitochondrial reactive oxygen species production. Exp Ther Med 2015;10:1224.

19 Wang WZ, Yao XD, Huang XJ, Li JQ Xu H: Effects of TGF- $\beta 1$ and alginate on the differentiation of rabbit bone marrow-derived mesenchymal stem cells into a chondrocyte cell lineage. Exp Ther Med 2015;10:995.

20 He QQ He X, Wang YP, Zou Y, Xia QJ, Xiong LL, Luo CZ, Hu XS, Liu J, Wang TH: Transplantation of bone marrow-derived mesenchymal stem cells (BMSCs) improves brain ischemia-induced pulmonary injury in rats associated to TNF- $\alpha$ expression. Behav Brain Funct 2016;12:1-14.

-21 Liu S, Zhang E, Yang M, Lu L: Overexpression of Wnt11 promotes chondrogenic differentiation of bone marrow-derived mesenchymal stem cells in synergism with TGF- $\beta$. Mol Cell Biochem 014;390:123-131.

$\checkmark 22$ Livak KJ, Schmittgen TD: Analysis of relative gene expression data using real-time quantitative PCR and the 2(-Delta Delta C(T)) Method. Methods 2001;25:402-408.

23 Casado-Díaz A, Pérez GD, Quesada-Gómez JM: 5.36 - Stem Cell Research and Molecular Markers in Medicine. Comprehensive Biotechnology:second Edition 2011;11:455-466.

24 Yoo JU, Barthel TS, Nishimura K, Solchaga L, Caplan AI, Goldberg VM, Johnstone B: The chondrogenic potential of human bone-marrow-derived mesenchymal progenitor cells. J Bone Joint Surg Am 1998;80:1745-1757.

25 Nakahara H, Dennis JE, Bruder SP, Haynesworth SE, Lennon DP, Caplan AI: In vitro differentiation of bone and hypertrophic cartilage from periosteal-derived cells. Exp Cell Res 1991;195:492.

26 Park MS, Kim YH, Jung Y, Kim SH, Park JC, Yoon DS, Kim SH, Lee JW: In situ recruitment of human BMSCs using chemokines for articular cartilage regeneration. Cell Transplant 2014;24:

-27 Kwok C, Weller PA, Guioli S, Foster JW, Mansour S, Zuffardi O, Punnett HH, Dominguez-Steglich MA, Brook JD, Young ID: Mutations in SOX9, the gene responsible for Campomelic dysplasia and autosomal sex reversal. Am J Hum Genet 1995;57:1028. 
Jiang et al.: Roles of Vitamin D and TGF- $\beta 1$ in BMSCs

28 Ikegami D, Akiyama H, Suzuki A, Nakamura T, Nakano T, Yoshikawa H, Tsumaki N: Sox9 sustains chondrocyte survival and hypertrophy in part through Pik3ca-Akt pathways. Development 2011;138:1507.

29 Zhao Q Eberspaecher H, Lefebvre V, De CB: Parallel expression of Sox9 and Col2a1 in cells undergoing chondrogenesis. Dev Dynam 1997;209:377-386.

-30 Bezancenet S: Correlation of COL10A1 induction during chondrogenesis of mesenchymal stem cells with demethylation of two CpG sites in the COL10A1 promoter. Arthritis Rheumatol 2008;58:2743-2753.

-31 Ansar MM, Esfandiariy E, Mardani M, Hashemibeni B, Zarkesh-Esfahani SH, Hatef M, Kabiri A: A comparative study of aggrecan synthesis between natural articular chondrocytes and differentiated chondrocytes from adipose derived stem cells in 3D culture. Adv Biomed Res 2012;1:24.

-32 Lu Z, Wang L, Pan H, Lin X, Lin C, Liu B, Zheng L, Zhao J: Stimulating Effect of a Newly Synthesized Sulfonamido-Basedgallate on Articular Chondrocytes in Vitro. Cell Physiol Biochem 2015;37:1196-1209.

33 Kim EK, Choi EJ: Compromised MAPK signaling in human diseases: an update. Arch Toxicol 2015;89:867882.

34 Zhang Y, Wang J, Lv Z, Zhao D, Luo M: Cox-2 promotes mesenchymal stem cells differentiation into cardiocytes by activating JNK and ERK pathway. Biochem Bioph Res Commun 2016;480:101-105. 